\title{
Topical anesthesia therapy using lidocaine-loaded nanostructured lipid carriers: tocopheryl polyethylene glycol 1000 succinate-modified transdermal delivery system
}

This article was published in the following Dove Medical Press journal:

Drug Design, Development and Therapy

\section{Xiangju Zhao \\ Ying Sun \\ Zhaoguo Li}

Department of Anesthesiology, Affiliated Hospital of Jining Medical University, Jining, Shandong 272000, People's Republic of China
Correspondence: Ying Sun Department of Anesthesiology, Affiliated Hospital of Jining Medical University, No 89 Guhuai Road, Jining, Shandong Province 272000, People's Republic of China

Tel +8605372903019

Email sunyingjnmu@I63.com
Purpose: Transdermal drug delivery of local anesthetics using lipid nanoparticles could enhance lipophilic drugs permeation through the stratum corneum, improve drug diffusion to deeper skin, and exert good therapeutic effects. The purpose of this study was to engineer a Tocopheryl Polyethylene Glycol 1000 Succinate (TPGS)-modified cationic nanostructured lipid carriers (NLC) for the delivery of lidocaine (LID; TPGS/LID-NLC).

Materials and methods: TPGS/LID-NLC was prepared by solvent diffusion method. The particle size, polydispersity index, zeta potential, drug entrapment efficiency, drug loading, stability, drug release, and cytotoxicity were tested to evaluate the basic characters of NLC. In vitro skin permeation and in vivo anesthesia effect in an animal model were further investigated to determine the therapeutic efficiency of the system.

Results: TPGS/LID-NLC had a particle size of $167.6 \pm 4.3 \mathrm{~nm}$, a zeta potential of $+21.2 \pm 2.3 \mathrm{mV}$, an entrapment efficiency of $85.9 \% \pm 3.1 \%$, and a drug loading of $11.5 \% \pm 0.9 \%$. A sustained release pattern was achieved by TPGS/LID-NLC, with $81.2 \%$ of LID released at 72 hours. In vitro permeation study showed that the steady-state fluxes $\left(\mathrm{J}_{\mathrm{ss}}\right)$, permeability coefficient $(\mathrm{Kp})$, and cumulative drug permeation $Q_{n}$ at 72 hours $\left(Q_{72}\right)$ of TPGS/LID-NLC were $15.6 \pm 1.8 \mu \mathrm{g} / \mathrm{cm}^{2} /$ hour, $10.3 \pm 0.9 \mathrm{~cm} /$ hour $\left(\times 10^{-3}\right)$, and $547.5 \pm 23.6 \mu \mathrm{g} / \mathrm{cm}^{2}$, respectively, which were significantly higher than the nonmodified NLC and free drug groups. In vivo anesthesia effect of TPGS/LID-NLC was the most remarkable and long acting among the formulations tested, which could be concluded by the most considerable maximum possible effect from 10 to 120 minutes during the whole research.

Conclusion: The most prominent in vitro permeation efficiency and in vivo anesthetic effect of TPGS/LID-NLC could be the evidence that TPGS-modified NLC could function as a promising drug delivery system for prolonged and efficient local anesthetic therapy.

Keywords: local anesthetics, transdermal delivery, tocopheryl succinate, nanostructured lipid carriers, lidocaine

\section{Introduction}

Topical anesthesia is one of the most applied strategies to reduce anxiety and pain caused by needle insertion and injection of local anesthetics. ${ }^{1-3}$ Transdermal drug delivery of local anesthetics brings further advantages like continuous drug delivery and fewer systemic adverse reactions. ${ }^{4,5}$ It is well known that local anesthetics applied to the skin permeate through intercellular pathway, namely through the lipid domains of the skin barrier (the stratum corneum). ${ }^{6}$ Unfortunately, the stratum corneum limits 
the permeability of local anesthetics and is the main obstacle for their clinical application. ${ }^{7}$ Most commercially available topical anesthetics consist of either lidocaine (LID) or prilocaine in lipid-soluble free base form, such as EMLA Cream (LID 2.5\% and prilocaine $2.5 \%$ ). ${ }^{8}$ However, EMLA is currently underutilized because of its slow efficacy and wide variation in onset and efficacy. ${ }^{9}$ Thus, it is urgent to design an alternative drug delivery system that can enhance both the retention time and the distribution of local anesthetics, finally achieving rapid, effective, and prolonged local transdermal anesthesia.

Lipid nanoparticles could enhance the lipophilic drugs permeation through the stratum corneum. ${ }^{10,11}$ It has been known that when the lipophilic drugs (such as LID and prilocaine) are encapsulated in lipid-based nanoparticles, their permeation through the skin is enhanced. ${ }^{10}$ Local anesthetics incorporated into lipid-based nanoparticles have also been reported to result in better retention of LID in the skin layers and show greater effectiveness as well as reduction in side effects. ${ }^{11}$ Thus, much attention has been paid to develop suitable lipid nanoparticles for topical administration. Nanostructured lipid carriers (NLC), the second generation of lipid nanoparticles, were emerged at the turn of the millennium and have evolved from solid lipid nanoparticles (SLN). ${ }^{12,13}$ Compared with SLN, NLC have merits such as higher drug loading (DL) and lower drug leakage during storage because of their compositions, which were composed of solid and liquid lipids. ${ }^{14}$ As for topical administration, NLC have chemical similarity to skin lipids, being therefore more permeable, well tolerated, biodegradable, and nontoxic. ${ }^{12}$ Topical applications of aqueous NLC dispersions are known to create an occlusive thin lipid film onto the skin tissue, which avoids water evaporation, and thus increases the skin's moisture and hydration, as well as enable controlled drug release. ${ }^{15,16}$ Cationic NLC can be beneficial in interacting with the negatively charged skin surface, thus improving drug diffusion to deeper skin and exerting therapeutic effects. ${ }^{17-20}$ In this study, a positively charged lipid, stearic acid (SA), was chosen to incorporate in the oil-lipid blend.

Tocopheryl Polyethylene Glycol 1000 Succinate (TPGS), a water-soluble derivative of natural vitamin $\mathrm{E}$, has been approved by FDA as a pharmaceutical ingredient. ${ }^{21}$ Recently, TPGS has been widely used in developing various drug delivery systems. TPGS is considered safe in skin studies. ${ }^{22}$ In the studies of topical administration, TPGS was reported to enhance the solubility and percutaneous penetration of drugs such as estradiol, ibuprofen, and minoxidil, and to improve their therapeutic effects with minor systemic side effects. ${ }^{23-26}$
In the present study, TPGS-modified cationic NLC (TPGS-NLC) was engineered. LID was encapsulated into TPGS-NLC to get TPGS/LID-NLC. The obtained NLCs were characterized by determination of their particle size, zeta potential, drug encapsulation efficiency, and cytotoxicity, and then assessed for in vitro skin permeation and in vivo anesthesia effect.

\section{Materials and methods Materials}

Lidocaine, phosphatidic acid (PA) sodium salt from egg yolk lecithin (purity $>98 \%$ ), SA, polyoxyl 35 castor oil (CO), dimethyldioctadecylammonium bromide (DDAB), glycerin monostearate (GMS), hexadecyltrimethylammonium bromide, and MTT were purchased from Sigma Aldrich (St Louis, MO, USA). TPGS was obtained from Eastman Chemical Company (Kingsport, TN, USA). All other chemicals were of analytical grade or high-performance liquid chromatography (HPLC) grade and used without further purification.

\section{Preparation of NLC}

TPGS/LID-NLC (Figure 1) was prepared by solvent diffusion method. ${ }^{27} \mathrm{SA}(100 \mathrm{mg})$ and GMS (100 mg) were dispersed in $\mathrm{CO}(2 \mathrm{~mL})$ to from lipid dispersion. Lipid phase was prepared by dissolving PA (100 mg) and LID (100 mg) in $1 \mathrm{~mL}$ of dimethyl formamide and added to the lipid dispersion with heating at the temperature of $70^{\circ} \mathrm{C}-75^{\circ} \mathrm{C}$. TPGS (100 mg) and DDAB (1\%, w/v) were dispersed in Milli-Q water $(10 \mathrm{~mL})$ to form the aqueous phase. The aqueous phase was stirred at $800 \mathrm{rpm}$, the lipid phase was rapidly injected into it, and then continued to stir for 20 minutes. The resulting suspension was then dispersed with Milli-Q water and then dialyzed against for 24 hours to get TPGS/LID-NLC suspension. TPGS/LID-NLC suspension was filtered through a membrane with $0.45 \mu \mathrm{m}$ pore size and stored at $2^{\circ} \mathrm{C}-8^{\circ} \mathrm{C}$ before use.

TPGS-NLC (Figure 1) was prepared by the same method without adding LID.

LID-loaded NLC that does not contain TPGS (LIDNLC, Figure 1) was prepared by the same method without adding TPGS.

Free LID was prepared by dissolving LID $(100 \mathrm{mg})$ in Milli-Q water (10 mL).

\section{Characterization of NLC}

NLC suspension was diluted and measured using a Zetasizer (Zetasizer 3000; Malvern Instruments Co. Ltd., 


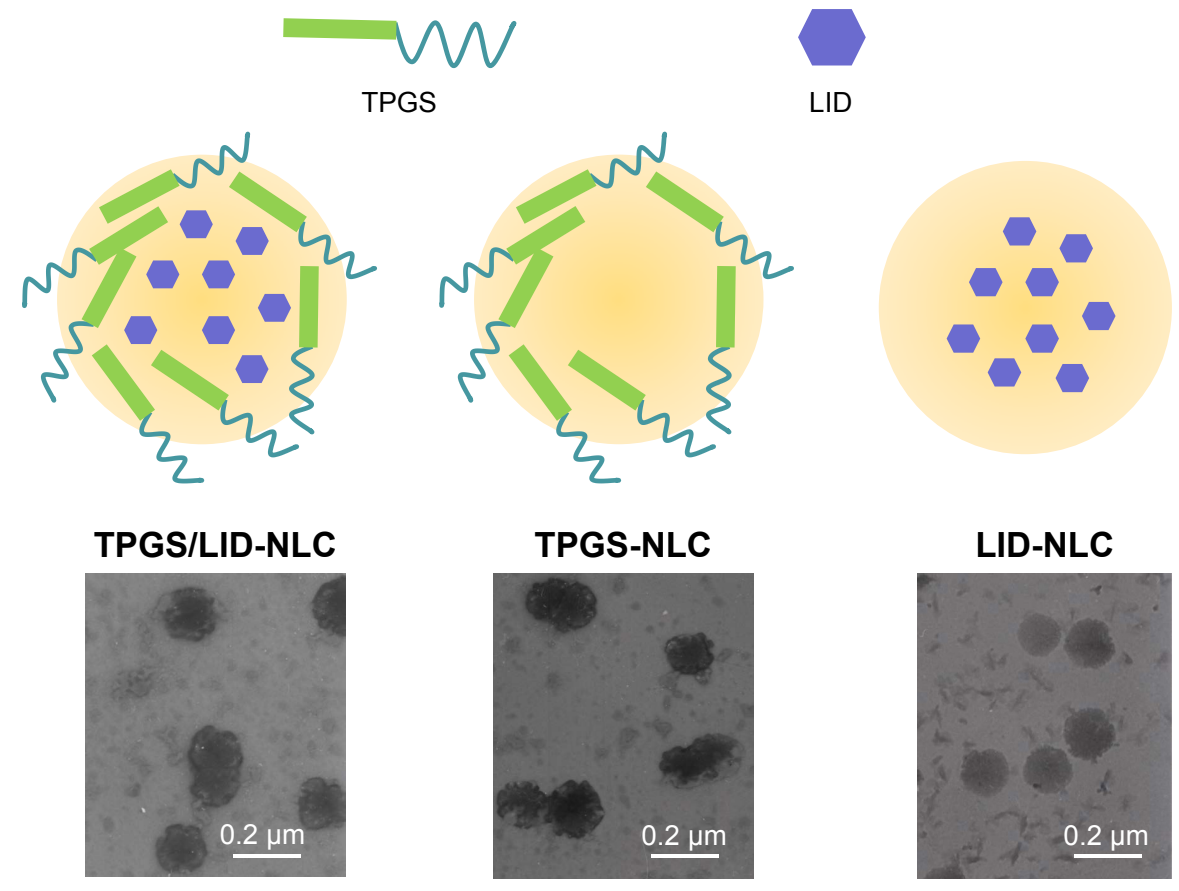

Figure I The scheme graph and TEM images of TPGS/LID-NLC, TPGS-NLC, and LID-NLC.

Notes: NLC was prepared by solvent diffusion method. The morphology of NLC was determined by TEM. A drop of NLC suspension was placed on the copper grid and equilibrated for 2 minutes. The liquid was wiped off with a filter paper, and samples were stained with phosphotungstic acid ( $2 \%$, w/v) for another 2.5 minutes and observed with TEM.

Abbreviations: LID, lidocaine; NLC, nanostructured lipid carriers; TEM, transmission electron microscopy; TPGS, tocopheryl polyethylene glycol I000 succinate.

Worcestershire, UK) to estimate the particle size, polydispersity index (PDI), and zeta potential. ${ }^{28}$ The morphology of NLC was determined by transmission electron microscopy (JEM-1200; JEOL Ltd., Tokyo, Japan). A drop of NLC suspension was placed on the copper grid and equilibrated for 2 minutes. The liquid was wiped off with a filter paper, and the samples were stained with phosphotungstic acid $(2 \%, \mathrm{w} / \mathrm{v})$ for another 2.5 minutes before observed. Entrapment efficiency (EE) and DL of TPGS/LID-NLC and LIDNLC were estimated using ultrafiltration centrifugation method. Briefly, NLC suspension (1 mL) was dissolved with methanol and sonicated (20 minutes) to destroy the structure. Then, the solution was centrifuged at 12,000 rpm for 20 minutes. Content of LID was measured by using HPLC, performed on an Agilent 1100 series with a zorbax RP-select B column, and a G1315A Diode-array detector (Agilent Technologies, Santa Clara, CA, USA) ${ }^{29}$ The EE and DL were calculated using the following equations:

$\mathrm{EE} \%=$

(Total amount of LID - Amount of free LID) $\times 100 \%$ (Total amount of LID)

$\mathrm{DL} \%=$

$\frac{(\text { Total amount of LID }- \text { Amount of free LID) }}{(\text { Total amount of lipid })} \times 100 \%$.
Stability of TPGS/LID-NLC and LID-NLC was determined at three kinds of conditions at 1 month: $\left.:{ }^{30} 1\right) 4^{\circ} \mathrm{C} \pm 2{ }^{\circ} \mathrm{C}$, 2) room temperature $\left(25^{\circ} \mathrm{C} \pm 2^{\circ} \mathrm{C}\right)$, and 3$) 45^{\circ} \mathrm{C} \pm 2^{\circ} \mathrm{C}$. An aliquot of NLC suspensions was filled into transparent vials and placed in the abovementioned three conditions for 1 month. At 1, 2, 3, and 4 weeks, the samples were taken out and assayed for the content of LID by the method described in this section.

\section{In vitro drug release of NLC}

In vitro drug release studies were performed to investigate the release behavior of LID from the NLC by dialysis method. ${ }^{31}$ Briefly, TPGS/LID-NLC ( $1 \mathrm{~mL})$ and LID-NLC $(1 \mathrm{~mL})$ were sealed into a semipermeable dialysis membrane (molecular weight cutoff of $50 \mathrm{kDa}$ ). The dialysis bags were immersed in the PBS (pH 7.4), and incubated at $37^{\circ} \mathrm{C}$ with continuous stirring. Determined amount of samples $(0.5 \mathrm{~mL})$ were collected from the dissolution media at predetermined time intervals $(0.5,1,2,4,8,16,24,48$, and 72 hours), and the same amount of fresh buffer solution $(0.5 \mathrm{~mL})$ was added. The samples were analyzed for the release amount of LID by the method described in Characterization of NLC section.

\section{Cells}

Mouse embryonic fibroblasts cells (BALB/c-3T3 cells) were obtained from the American Type Culture Collection 
(Manassas, VA, USA) and cultured in DMEM supplemented with 10\% heat-inactivated fetal bovine serum (Gibco, Waltham, MA, USA) at $37^{\circ} \mathrm{C}$ using a humidified $5 \% \mathrm{CO}_{2}$ incubator.

\section{In vitro cytotoxicity of NLC}

In vitro cytotoxicity of the NLC was evaluated using BALB/c3 T3 cells by MTT assay. ${ }^{32}$ Samples of TPGS/LID-NLC, TPGS-NLC, LID-NLC, and free LID were prepared with the concentrations ranging from 0.1 to $10 \mathrm{mg} / \mathrm{mL}$. BALB/c-3T3 cells $\left(1 \times 10^{5}\right)$ were seeded into 96 -well plates and incubated for 48 hours. Different concentrations of samples were added to each well and incubated for 24 hours. Cells were then detected by incubation in the presence of MTT solution $(25 \mu \mathrm{L}, 5 \mathrm{mg} / \mathrm{mL})$ for 2 hours at $37^{\circ} \mathrm{C}$. The amount of MTT converted to formazan was measured to determine the percentage of viable cells, using a microplate reader (Bio-Rad, Hercules, CA, USA) at a wavelength of $630 \mathrm{~nm}$.

\section{Animals}

Animal study protocol was approved by the Ethics Committee of Jining Medical University and followed the National Institutes of Health guide for the care and use of Laboratory animals (NIH publications no. 8023). Female SpragueDawley rats (9-11 weeks, 220-240 g) were purchased from Shandong University Laboratory Animal Center and were kept under standard laboratory conditions (temperature, $25^{\circ} \mathrm{C} \pm 2{ }^{\circ} \mathrm{C}$; relative humidity, $55 \% \pm 5 \% ; 12 / 12$ hour light/ dark cycle).

\section{In vitro permeation efficiency of NLC}

Sprague-Dawley rats were anesthetized and excised the abdominal full-thickness skin. ${ }^{33}$ The hair, muscle, fat, and vasculature on the skin were removed. After washing and equilibrating with PBS buffer, the stripped skin was tied on static vertical Franz Diffusion cells (PermeGear Inc., Bethlehem, PA, USA) with a donor area of $0.64 \mathrm{~cm}^{2}$ and a receptor volume of $5.1 \mathrm{~mL} .^{34}$ The receptor compartment was filled with PBS ( $\mathrm{pH} 7.4)$, which was maintained at $37^{\circ} \mathrm{C} \pm 0.5^{\circ} \mathrm{C}$ and constantly stirred at $400 \mathrm{rpm}$. TPGS/LIDNLC, LID-NLC, Xylocaine ${ }^{\circledR}$ (LID HCl) 2\% Jelly (Xylocaine Jelly), and free LID $(0.5 \mathrm{~mL})$ were added to the dorsal side of the mice skin. Samples $(0.3 \mathrm{~mL})$ were collected from the receptor compartment at predetermined time points $(0.5,1,2$, $4,8,16,24,48$, and 72 hours) and replaced with equivalent amount of PBS. The samples were analyzed for the permeate amount of LID by the method defined in Characterization of NLC section. The cumulative amount of LID penetrated $\left(Q_{n}\right)$ as a function of time was calculated using the following equations: ${ }^{35}$

$$
\mathrm{Q}_{\mathrm{n}}=\frac{\mathrm{C}_{\mathrm{n}} \times \mathrm{V}_{0}+\sum_{\mathrm{i}=0}^{\mathrm{n}-1} \mathrm{C}_{\mathrm{i}} \times \mathrm{V}_{\mathrm{i}}}{\mathrm{A}}
$$

where $\mathrm{C}_{\mathrm{n}}$ and $\mathrm{C}_{\mathrm{i}}$ refer to the drug concentration of the receptor medium at each sampling time and the drug concentration of the ith sample. $\mathrm{V}_{0}$ and $\mathrm{V}_{\mathrm{i}}$ correspond to the volumes of the receptor compartment and the collected sample. A refers to the effective diffusion area. The steady-state fluxes $\left(\mathrm{J}_{\mathrm{ss}}\right)$ were obtained from the slope of the linear region of the curve, representing the amount of drug permeated per unit area vs time. Based on the $\mathrm{J}_{\mathrm{ss}}$, the permeability coefficient (Kp) was estimated according to the following equation:

$$
\mathrm{J}_{\mathrm{ss}}=\mathrm{C}_{0} \mathrm{Kp}
$$

where $\mathrm{C}_{0}$ corresponds to the drug concentration in the donor compartment.

\section{In vivo anesthesia effect of NLC}

The tail-flick (TF) test is the most frequently used method to measure pain levels and assess the effects of anesthesia. ${ }^{36}$ Rats were placed in a plastic box and the ventral surface of the distal 5-6 cm of the tail was placed over a $0.5-\mathrm{cm}$ hole in an aluminum box, and an infrared radiant (IR) bulb was placed beneath the hole. TPGS/LID-NLC, LID-NLC, Xylocaine Jelly, free LID (containing $2 \mathrm{mg}$ of LID each), and $0.9 \%$ saline solution (as control) were applied onto the dorsal surface of the tail. The tail was exposed to the IR bulb at a distance of $5 \mathrm{~mm}$ in each trial, and a 10 -second cutoff was used to minimize the risk of tissue damage (cutoff latency). Baseline latencies were typically ranged from 2.5 to 3.0 seconds. In vivo anesthesia effect could be evaluated using the maximum possible effect (MPE) calculated by TF latency and represented by the following equation:

$$
\operatorname{MPE}(\%)=\frac{(\text { Test latency }- \text { Baseline latency })}{(\text { Cutoff latency }- \text { Baseline latency })} \times 100
$$

\section{Statistical analysis}

All studies were repeated at least three times. Results were reported as mean \pm standard deviation. Statistical significance was tested by one-way analysis of variance, 
with $P$-values $<0.05$ indicating statistically significant differences.

\section{Results}

\section{Characterization of NLC}

The image of the LID-NLC revealed spherical shape nanoparticles without hierarchy (Figure 1). The images of TPGS/ LID-NLC and TPGS-NLC had obvious outer layers on the surface of particles. The sizes of TPGS/LID-NLC, TPGSNLC, and LID-NLC were smaller than $200 \mathrm{~nm}$ according to the $0.2 \mu \mathrm{m}$ bars in the images. The mean particle size, PDI, and zeta potential are listed in Table 1. The sizes of TPGS/

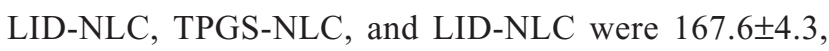
$163.9 \pm 3.8$, and $148.8 \pm 2.9 \mathrm{~nm}$, respectively. The PDI of all the NLCs ranged from 0.121 to 0.156 . EE is calculated as the percentage ratio between weight of drug loaded into NLC and the drug added during preparation. The EEs of the TPGS/LID-NLC and LID-NLC were $85.9 \% \pm 3.1 \%$ and $87.1 \% \pm 2.6 \%$, respectively. DL is defined as the amount of drug in comparison with the lipid materials used for the NLC preparation. The DL of TPGS/LID-NLC was lower than that of LID-NLC, which may be explained by the addition of TPGS that increased the amount of the ingredients. Both TPGS/LID-NLC (Figure 2A) and LID-NLC (Figure 2B) showed high stability at $4{ }^{\circ} \mathrm{C}$ with the percentage of LID remaining $>80 \%$ at 4 weeks, whereas the remaining LID was lower at the end of 1 month at $25^{\circ} \mathrm{C}$ and at $45^{\circ} \mathrm{C}(P<0.05)$. The results revealed that the NLC should be kept at low temperature until use and could be stable within 1 month.

\section{In vitro drug release}

As showed in Figure 3, LID release rates from TPGS/LIDNLC and LID-NLC are different. At 24 hours, LID-NLC have already completed $>80 \%$ of drug release, while the cumulative release parameter for TPGS/LID-NLC was only $61.3 \%$. TPGS/LID-NLC reached $81.2 \%$ of LID release at 72 hours. This may be the evidence for the existence of polyethylene glycol (PEG) molecule in TPGS introduced in a more sustained release to the system.

\section{In vitro cytotoxicity}

In vitro cytotoxicity of NLC was tested by evaluating BALB/c-3T3 cells viability (Figure 4). At the concentrations used, exposure of cells to drug free TPGS-NLC had no obvious changes of viability. LID-loaded TPGS/LID-NLC and LID-NLC showed a moderate effect on cellular viability, with values slowly decreasing from $90 \%$ to $80 \%$. Decreases in viability of free LID-treated group were found at high concentrations $(1,5$, and $10 \mathrm{mg} / \mathrm{mL}$ ), and reduced to $59 \%$ at the concentration of $10 \mathrm{mg} / \mathrm{mL}$.

\section{In vitro permeation efficiency}

In vitro permeation study of LID from NLC was carried out by using Franz diffusion cells with the skin of rats. The accumulative penetration amount $\left(Q_{n}\right)$ of LID from TPGS/ LID-NLC, LID-NLC, Xylocaine Jelly, and free LID is shown in Figure 5. The steady-state fluxes $\left(\mathrm{J}_{\mathrm{ss}}\right)$, permeability coefficient $(\mathrm{Kp})$ and $\mathrm{Q}_{\mathrm{n}}$ at $24\left(\mathrm{Q}_{24}\right), 48\left(\mathrm{Q}_{48}\right)$, and 72 hours $\left(\mathrm{Q}_{72}\right)$ are summarized in Table 2. It was evident that LID-NLC significantly increased the $\mathrm{J}_{\mathrm{ss}}$ of LID to $10.6 \pm 1.3 \mu \mathrm{g} / \mathrm{cm}^{2} /$ hour when compared with free LID $\left(6.8 \pm 0.7 \mu \mathrm{g} / \mathrm{cm}^{2} /\right.$ hour; $\left.P<0.05\right)$. TPGS/LID-NLC exhibited the most prominent $\mathrm{J}_{\mathrm{ss}}$ of $15.6 \pm 1.8 \mu \mathrm{g} / \mathrm{cm}^{2} /$ hour, better than Xylocaine Jelly $(P<0.05)$. $\mathrm{Q}_{72}$ of TPGS/LID-NLC, LID-NLC, Xylocaine Jelly, and free LID were $547.5 \pm 23.6,383.7 \pm 13.96,413.8 \pm 27.6$, and $254.8 \pm 9.7 \mu \mathrm{g} / \mathrm{cm}^{2}$, respectively. This tendency indicated that the potential of NLC in substantially delivering LID across skin was considerable, relative to the free solution.

\section{In vivo anesthesia effect}

TF test was conducted to analyze the local anesthesia in the tail of the rat (Figure 6). The results suggest that all formulations showed fast anesthesia effect at 10 minutes of the study. However, NLC formulas exhibit higher efficiency than free LID with higher MPE $(P<0.05)$. On the other hand, the conventional marketed jelly formulation had a fast onset of action and the skin was totally anesthetized. Local anesthesia ability of NLCs lasted longer than Xylocaine

Table I Particle size, PDI, zeta potential, EE, and DLC of NLC (mean $\pm S D, n=3$ )

\begin{tabular}{|l|l|l|l|l|l|}
\hline Formulation & Particle size $\mathbf{( n m})$ & PDI & Zeta potential $(\mathbf{m V})$ & EE $(\%)$ & DL $(\%)$ \\
\hline TPGS/LID-NLC & $167.6 \pm 4.3$ & $0.156 \pm 0.026$ & $+21.2 \pm 2.3$ & $85.9 \pm 3.1$ & $11.5 \pm 0.9$ \\
\hline TPGS-NLC & $163.9 \pm 3.8$ & $0.137 \pm 0.015$ & $+23.5 \pm 2.8$ & - & - \\
\hline LID-NLC & $148.8 \pm 2.9$ & $0.121 \pm 0.012$ & $+34.7 \pm 3.6$ & $87.1 \pm 2.6$ & $18.2 \pm 1.1$ \\
\hline
\end{tabular}

Abbreviations: DLC, drug-loading capacity; EE, entrapment efficiency; LID, lidocaine; NLC, nanostructured lipid carriers; PDI, polydispersity index; TPGS, tocopheryl polyethylene glycol 1000 succinate. 

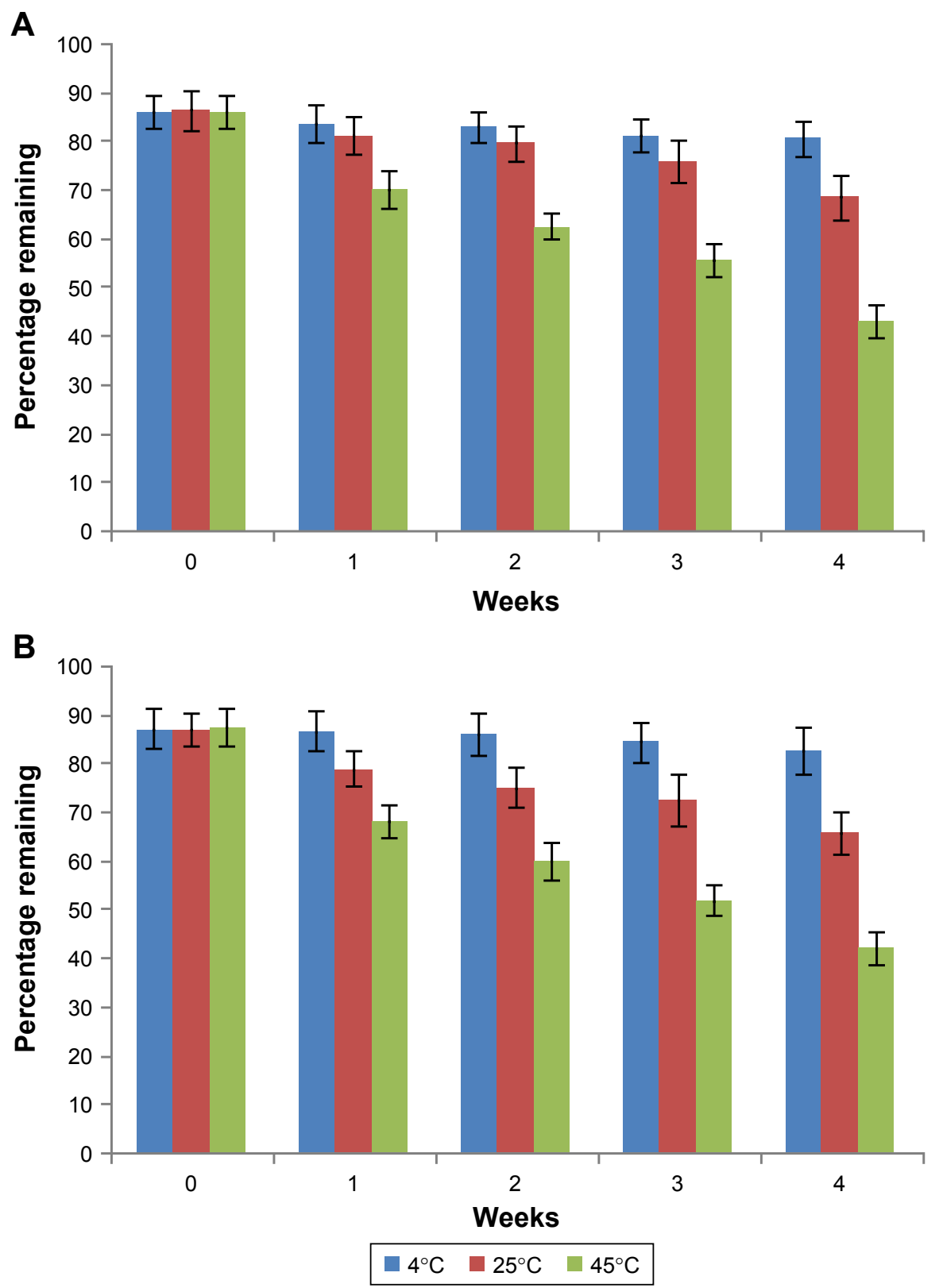

Figure 2 Stability of TPGS/LID-NLC (A) and LID-NLC (B) determined at three temperature conditions at I month. Data represent the mean \pm SD, $n=3$.

Notes: An aliquot of NLC suspensions was filled into transparent vials and placed in the abovementioned three conditions for I month. At I, 2 , 3, and 4 weeks, the samples were taken out and assayed.

Abbreviations: LID, lidocaine; NLC, nanostructured lipid carriers; TPGS, tocopheryl polyethylene glycol 1000 succinate.

Jelly and free LID $(P<0.05)$. TPGS/LID-NLC showed the most remarkable and long-acting anesthesia ability among the three formulations, which could be concluded by the most considerable MPE from 10 to 120 minutes during the whole research.

\section{Discussion}

In the present study, TPGS-modified cationic NLC (TPGSNLC) was designed and applied for the topical anesthesia.
TPGS is formed by esterification of vitamin E succinate with $\mathrm{PEG}_{1000}$. TPGS has served as a permeation enhancer. ${ }^{37}$ The particle size results indicate that the use of TPGs did increase the size of the NLC: TPGS/LID-NLC (167.6 nm) leads to a significant increase in the size in comparison to LID-NLC $(148.8 \mathrm{~nm}) \cdot{ }^{38}$ PDI values provide an indication of the degree of homogeneity in a system, with values in the range $0.1-0.2$ reflecting a monodisperse nanoparticle distribution. ${ }^{39}$ The PDI values for the NLC formulations 


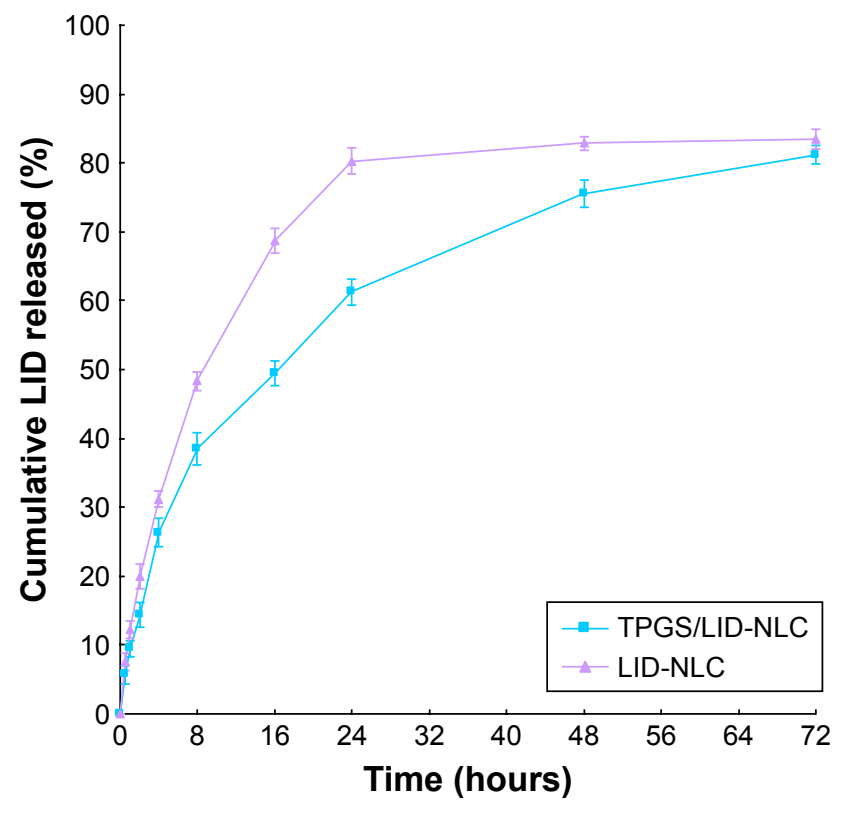

Figure 3 In vitro cumulative LID release from TPGS/LID-NLC and LID-NLC. Data represent the mean $\pm S D, n=3$.

Notes: The dialysis bags were immersed in the PBS $(\mathrm{pH} 7.4)$ and incubated at $37^{\circ} \mathrm{C}$ with continuous stirring. Determined amount of samples $(0.5 \mathrm{~mL})$ were collected from the dissolution media and analyzed by HPLC.

Abbreviations: HPLC, high-performance liquid chromatography; LID, lidocaine; NLC, nanostructured lipid carriers; TPGS, tocopheryl polyethylene glycol 1000 succinate.

were in the range $0.121-0.156$. Zeta potential values provide valuable information about the stability of a colloidal system, and can be influenced by factors including the electrical conductivity and the nature of the reagents.
The positive value of Zeta potential of NLC can be attributed to the presence of DDAB used in the formula, which is a double-tailed cationic lipid. ${ }^{40}$ Drug encapsulation efficiency is calculated as the percentage ratio between the amount of drug loaded in NLC and the amount of the total drug added during fabrication. EE of TPGS/LID-NLC and LID-NLC was found to be $85.9 \%$ and $87.1 \%$, which indicates the NLCs were highly effective carriers for the encapsulation of LID.

Stability of NLCs was determined at $4^{\circ} \mathrm{C}, 25^{\circ} \mathrm{C}$, and $45^{\circ} \mathrm{C}$. Both TPGS/LID-NLC and LID-NLC showed a high stability at $4^{\circ} \mathrm{C}$ within 1 month. However, at the two higher temperatures, the remaining LID was higher in TPGS/LIDNLC than in LID-NLC. This phenomenon may be explained by the PEG chains of TPGS which could stretch in the outer layer of the carriers, and thus could prevent the aggregation between the particles, affecting the packing and storage conditions of formulations. ${ }^{41}$

NLC systems have significantly prolonged the release of LID. A drug is usually released from an appropriate drug delivery system and its therapeutic activity is exerted after its entrance into the target site. ${ }^{42}$ The sustained release of LID from NLC might be due to the diffusion of LID from NLC and the degradation or hydrolysis of NLC..$^{43}$ The LID release rate from TPGS/LID-NLC was more sustained than LID-NLC. This could be the evidence that the modification of TPGS provides a more sustained drug release to the NLC

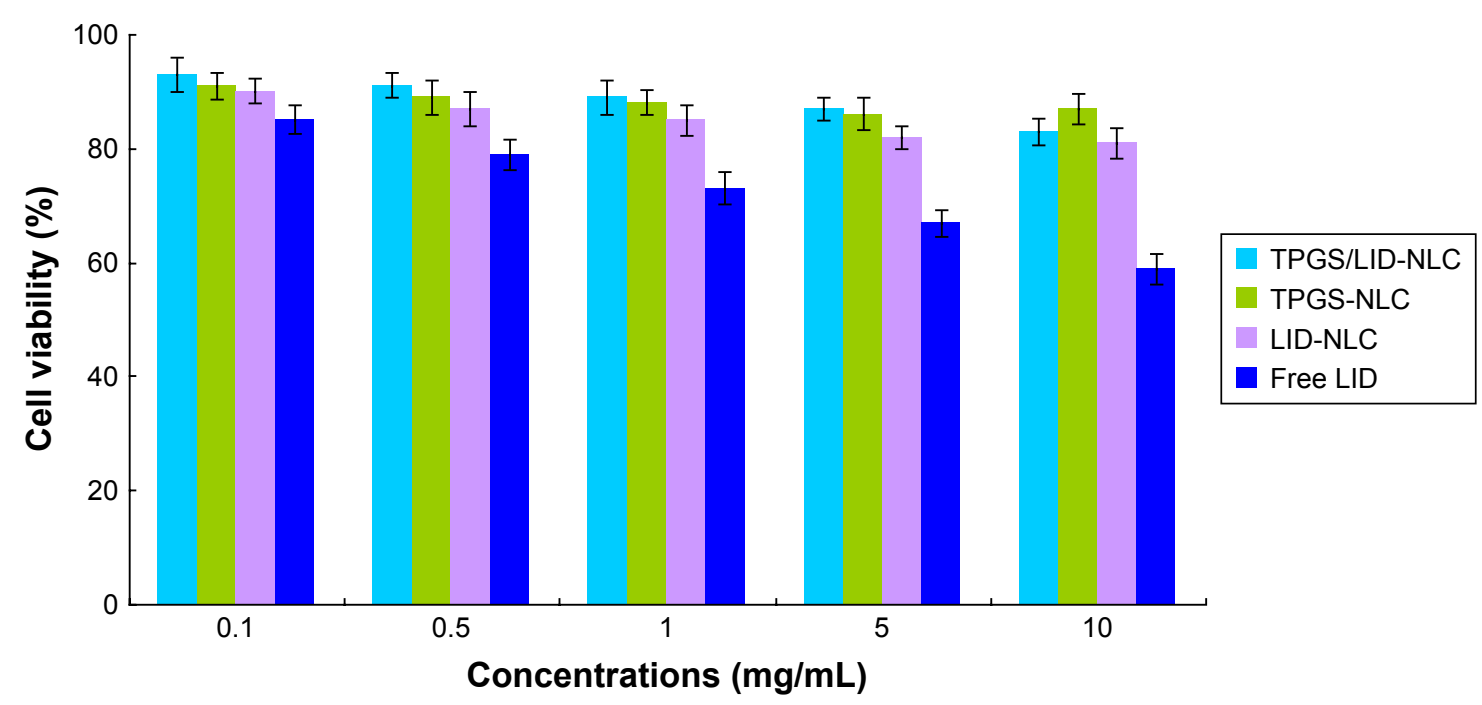

Figure 4 In vitro cytotoxicity of TPGS/LID-NLC, TPGS-NLC, LID-NLC, and free LID tested by evaluating BALB/c-3T3 cells viability. Data represent the mean \pm SD, $n=6$. Notes: Different concentrations of samples were added to each well and incubated for 24 hours. Cells were then detected by incubation in the presence of MTT solution $\left(25 \mu \mathrm{L}, 5 \mathrm{mg} / \mathrm{mL}\right.$ ) for 2 hours at $37^{\circ} \mathrm{C}$. The amount of MTT converted to formazan was measured to determine the percentage of viable cells, using a microplate reader at a wavelength of $630 \mathrm{~nm}$.

Abbreviations: LID, lidocaine; NLC, nanostructured lipid carriers; TPGS, tocopheryl polyethylene glycol I,000 succinate. 


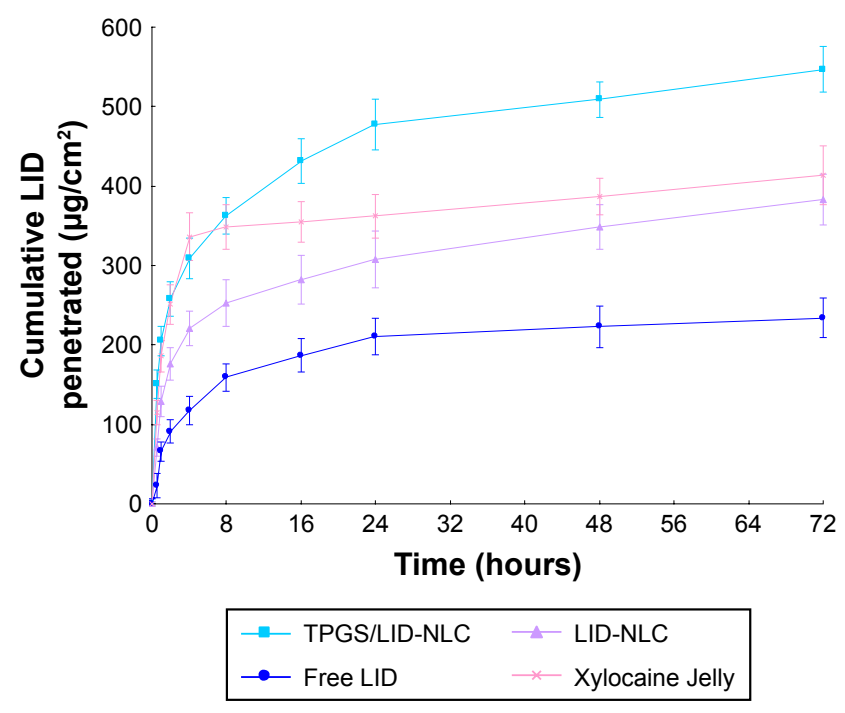

Figure 5 In vitro drug permeation behaviors of LID from TPGS/LID-NLC, LIDNLC, and free LID accounted by the accumulative penetration amount $\left(Q_{n}\right)$ of LID. Data represent the mean $\pm S D, n=6$.

Notes: TPGS/LID-NLC, LID-NLC, Xylocaine Jelly, and free LID were added to the dorsal side of the mice skin. Samples were collected from the receptor compartment and analyzed by HPLC.

Abbreviations: HPLC, high-performance liquid chromatography; LID, lidocaine; NLC, nanostructured lipid carriers; TPGS, tocopheryl polyethylene glycol 1000 succinate.

system, which may help with the long-lasting anesthesia effect. In vitro cytotoxicity of NLC was carried out on BALB/c-3T3 cells. The cell viability of NLCs over the studied concentration range was between $80 \%$ and $100 \%$, compared with the control. TPGS/LID-NLC showed no higher cytotoxicity than LID-NLC at all concentrations. On the contrary, free LID-treated group found at high concentrations exhibited obvious cytotoxicity.

The permeation enhancing ability of NLC for topical delivery could be explained by the nanocarrier properties. ${ }^{44}$ Small sizes of NLCs maintain close contact with the stratum corneum, thus increasing the amount of encapsulated drugs into the skin. The phospholipid molecules are reported to get incorporated in the skin layers, fluidize, and loosen the lipidic matrix of the skin, thus promoting the drug permeation. ${ }^{45}$ So in this study, the kinds of lipid used in the formulation can help in improving the skin permeation of the LID. The permeation study of LID from NLC was carried out by using Franz diffusion cells with the skin of rats. LID-NLC significantly increased the $\mathrm{J}_{\mathrm{ss}}$ of LID from the free LID. Taking into consideration the highest $\mathrm{J}_{\mathrm{ss}}, \mathrm{Kp}$, and $\mathrm{Q}_{\mathrm{n}}$ of TPGS/LID-NLC, it may be the most suitable system for the drug permeation, whose efficiency needs to be further determined by the in vivo studies.

To evaluate the effects of anesthesia in vivo, TF test is most frequently used. ${ }^{46}$ Other in vivo infringement stimulation involves pinprick, forceps pinch, and so on, but these types of methods may damage the skin of the animal and possibly lead to an increasing difficulty in evaluating the effect of the anesthesia. ${ }^{47}$ The saline group showed no obvious change of MPE during the experiment. The conventional marketed jelly formulation had a fast onset of action and the skin was totally anesthetized. This indicated that the ionized form of LID penetrated much more rapidly than the free base but was rapidly absorbed and cleared off by the cutaneous capillaries. ${ }^{11}$ NLC formulas exhibited higher and longer anesthesia efficiency than free LID with higher MPE until 120 minutes of the study. TPGS/LID-NLC showed the most remarkable and long-acting anesthesia ability among the three formulations, which demonstrated that TPGS modification promoted the skin delivery of the loaded drug and achieved the longest duration of TF test; also the long-lasting effect may due to the TPGS on the NLC surface would release the drug slowly thereby acting for a longer period of time.

\section{Conclusion}

TPGS/LID-NLC was fabricated and applied for the topical anesthesia using LID as the payload. In vitro and in vivo permeation efficiency and anesthetic effect of TPGS/LIDNLC were better than nonmodified LID-NLC and free LID. These could be the evidence that TPGS-modified NLC could function as a promising drug delivery system for prolonged and prominent local anesthetic effect with low toxicity.

Table 2 In vitro LID permeation parameters of NLC (mean $\pm S D, n=3$ )

\begin{tabular}{|l|l|l|l|l|l|}
\hline Formulation & $\mathbf{J}_{\mathrm{ss}}\left(\mu \mathrm{g} / \mathrm{cm}^{2} /\right.$ hour $)$ & $\mathbf{K p}(\mathbf{c m} / \mathbf{h o u r})\left(\times 10^{-3}\right)$ & $\mathbf{Q}_{24}\left(\mu \mathrm{g} / \mathrm{cm}^{2}\right)$ & $\mathbf{Q}_{48}\left(\mu \mathrm{g} / \mathrm{cm}^{2}\right)$ & $\mathbf{Q}_{72}\left(\mu \mathrm{g} / \mathrm{cm}^{2}\right)$ \\
\hline TPGS/LID-NLC & $15.6 \pm 1.8$ & $10.3 \pm 0.9$ & $478.2 \pm 17.9$ & $509.6 \pm 21.2$ & $547.5 \pm 23.6$ \\
\hline LID-NLC & $10.6 \pm 1.3$ & $6.9 \pm 0.5$ & $308.9 \pm 11.7$ & $349.3 \pm 9.8$ & $383.7 \pm 13.9$ \\
\hline Free LID & $6.8 \pm 0.7$ & $4.6 \pm 0.3$ & $211.7 \pm 8.5$ & $223.4 \pm 10.7$ & $254.8 \pm 9.7$ \\
\hline Xylocaine Jelly & $11.8 \pm 1.9$ & $7.8 \pm 0.8$ & $362.2 \pm 18.1$ & $387.3 \pm 23.9$ & $413.8 \pm 27.6$ \\
\hline
\end{tabular}

Abbreviations: LID, lidocaine; NLC, nanostructured lipid carriers; TPGS, tocopheryl polyethylene glycol I000 succinate. 




Figure 6 In vivo anesthesia effects of TPGS/LID-NLC, LID-NLC, and free LID evaluated by TF latency test. Data represent the mean \pm SD, $n=8$.

Notes: TPGS/LID-NLC, LID-NLC, Xylocaine Jelly, free LID, and $0.9 \%$ saline solution were applied onto the dorsal surface of the tail. In vivo anesthesia effect could be evaluated using the maximum possible effect calculated by TF latency.

Abbreviations: LID, lidocaine; NLC, nanostructured lipid carriers; TF, tail flick; TPGS, tocopheryl polyethylene glycol I000 succinate.

\section{Disclosure}

The authors report no conflicts of interest in this work.

\section{References}

1. de Araújo DR, da Silva DC, Barbosa RM, et al. Strategies for delivering local anesthetics to the skin: focus on liposomes, solid lipid nanoparticles, hydrogels and patches. Expert Opin Drug Deliv. 2013;10(11): 1551-1563.

2. Franz-Montan M, Ribeiro LNM, Volpato MC, et al. Recent advances and perspectives in topical oral anesthesia. Expert Opin Drug Deliv. 2017;14(5):673-684.

3. Peptu C, Rotaru R, Ignat L, et al. Nanotechnology approaches for pain therapy through transdermal drug delivery. Curr Pharm Des. 2015; 21(42):6125-6139.

4. Wang Y, Wang S, Shi P. Transcriptional transactivator peptide modified lidocaine-loaded nanoparticulate drug delivery system for topical anesthetic therapy. Drug Deliv. 2016;23(9):3193-3199.

5. Chen C, You P. A novel local anesthetic system: transcriptional transactivator peptide-decorated nanocarriers for skin delivery of ropivacaine. Drug Des Devel Ther. 2017;11:1941-1949.

6. Godin B, Touitou E. Transdermal skin delivery: predictions for humans from in vivo, ex vivo and animal models. Adv Drug Deliv Rev. 2007; 59(11):1152-1161.

7. Alexander A, Dwivedi S, Ajazuddin GTK, et al. Approaches for breaking the barriers of drug permeation through transdermal drug delivery. $J$ Control Release. 2012;164(1):26-40.

8. Pino CJ, Scherer MA, Shastri VP. Investigation of the transdermal transport of charged local anesthetics in the presence of triterpene saponin glycosides. Drug Deliv Transl Res. 2014;4(2):131-138.

9. Lander JA, Weltman BJ, So SS. EMLA and amethocaine for reduction of children's pain associated with needle insertion. Cochrane Database Syst Rev. 2006;3(3):CD004236.

10. Zhang L, Wang J, Chi H, Wang S. Local anesthetic lidocaine delivery system: chitosan and hyaluronic acid-modified layer-by-layer lipid nanoparticles. Drug Deliv. 2016;23(9):3529-3537.
11. Pathak $P$, Nagarsenker M. Formulation and evaluation of lidocaine lipid nanosystems for dermal delivery. AAPS PharmSci Tech. 2009;10(3): 985-992.

12. Doktorovova S, Souto EB. Nanostructured lipid carrier-based hydrogel formulations for drug delivery: a comprehensive review. Expert Opin Drug Deliv. 2009;6(2):165-176.

13. Müller RH, Shegokar R, Keck CM. 20 years of lipid nanoparticles (SLN and NLC): present state of development and industrial applications. Curr Drug Discov Technol. 2011;8(3):207-227.

14. Puglia C, Sarpietro MG, Bonina F, Castelli F, Zammataro M, Chiechio S. Development, characterization, and in vitro and in vivo evaluation of benzocaine- and lidocaine-loaded nanostructrured lipid carriers. J Pharm Sci. 2011;100(5):1892-1899.

15. Wissing S, Lippacher A, Müller R. Investigations on the occlusive properties of solid lipid nanoparticles (SLN). J Cosmet Sci. 2001;52(5): 313-324.

16. Nguyen CN, Nguyen TTT, Nguyen HT, Tran TH. Nanostructured lipid carriers to enhance transdermal delivery and efficacy of diclofenac. Drug Deliv Transl Res. 2017;7(5):664-673.

17. Mao KL, Fan ZL, Yuan JD, et al. Skin-penetrating polymeric nanoparticles incorporated in silk fibroin hydrogel for topical delivery of curcumin to improve its therapeutic effect on psoriasis mouse model. Colloids Surf B Biointerfaces. 2017;160:704-714.

18. El-Badry M, Fetih G, Shakeel F. Comparative topical delivery of antifungal drug croconazole using liposome and micro-emulsion-based gel formulations. Drug Deliv. 2014;21(1):34-43.

19. Eroğlu İ, Azizoğlu E, Özyazıcı M, et al. Effective topical delivery systems for corticosteroids: dermatological and histological evaluations. Drug Deliv. 2016;23(5):1502-1513.

20. Wavikar P, Pai R, Vavia P. Nose to brain delivery of rivastigmine by in situ gelling cationic nanostructured lipid carriers: enhanced brain distribution and pharmacodynamics. J Pharm Sci. 2017;106(12): 3613-3622.

21. Zhang Z, Tan S, Feng SS. Vitamin E TPGS as a molecular biomaterial for drug delivery. Biomaterials. 2012;33(19):4889-4906. 
22. Wan T, Pan J, Long Y, et al. Dual roles of TPGS based microemulsion for tacrolimus: enhancing the percutaneous delivery and anti-psoriatic efficacy. Int J Pharm. 2017;528(1-2):511-523.

23. $\mathrm{Mu} \mathrm{L}$, Seow PH. Application of TPGS in polymeric nanoparticulate drug delivery system. Colloids Surf B Biointerfaces. 2006;47(1):90-97.

24. Sheu MT, Wu AB, Lin KP, Shen CH, Ho HO. Effect of tocopheryl polyethylene glycol succinate on the percutaneous penetration of minoxidil from water/ethanol/polyethylene glycol 400 solutions. Drug Dev Ind Pharm. 2006;32(5):595-607.

25. Sheu MT, Chen SY, Chen LC, Ho HO. Influence of micelle solubilization by tocopheryl polyethylene glycol succinate (TPGS) on solubility enhancement and percutaneous penetration of estradiol. J Control Release. 2003;88(3):355-368.

26. Brownlow B, Nagaraj VJ, Nayel A, Joshi M, Elbayoumi T. Development and in vitro evaluation of vitamin E-enriched nanoemulsion vehicles loaded with genistein for chemoprevention against UVB-induced skin damage. J Pharm Sci. 2015;104(10):3510-3523.

27. Zhang J, Xiao X, Zhu J, et al. Lactoferrin- and RGD-comodified, temozolomide and vincristine-coloaded nanostructured lipid carriers for gliomatosis cerebri combination therapy. Int $J$ Nanomedicine. 2018;13:3039-3051.

28. Gao X, Zhang J, Xu Q, Huang Z, Wang Y, Shen Q. Hyaluronic acidcoated cationic nanostructured lipid carriers for oral vincristine sulfate delivery. Drug Dev Ind Pharm. 2017;43(4):661-667.

29. Wang J, Zhang L, Chi H, Wang S. An alternative choice of lidocaineloaded liposomes: lidocaine-loaded lipid-polymer hybrid nanoparticles for local anesthetic therapy. Drug Deliv. 2016;23(4):1254-1260.

30. Manosroi J, Lohcharoenkal W, Götz F, Werner RG, Manosroi W, Manosroi A. Transdermal absorption and stability enhancement of salmon calcitonin by Tat peptide. Drug Dev Ind Pharm. 2013;39(4): 520-525.

31. Boakye CH, Patel K, Patel AR, et al. Lipid-based oral delivery systems for skin deposition of a potential chemopreventive DIM derivative: characterization and evaluation. Drug Deliv Transl Res. 2016;6(5): 526-539.

32. You P, Yuan R, Chen C. Design and evaluation of lidocaine- and prilocaine-coloaded nanoparticulate drug delivery systems for topical anesthetic analgesic therapy: a comparison between solid lipid nanoparticles and nanostructured lipid carriers. Drug Des Devel Ther. 2017; 11:2743-2752.

33. Zhang Y, Yue Y, Chang M. Local anaesthetic pain relief therapy: in vitro and in vivo evaluation of a nanotechnological formulation co-loaded with ropivacaine and dexamethasone. Biomed Pharmacother. 2017;96: 443-449.

34. Ghosh I, Michniak-Kohn B. Design and characterization of submicron formulation for a poorly soluble drug: the effect of Vitamin E TPGS and other solubilizers on skin permeability enhancement. Int J Pharm. 2012;434(1-2):90-98.
35. Vitorino C, Almeida A, Sousa J, et al. Passive and active strategies for transdermal delivery using co-encapsulating nanostructured lipid carriers: in vitro vs. in vivo studies. Eur J Pharm Biopharm. 2014;86(2): 133-144.

36. Ouchi K, Sekine J, Koga Y, Nakao S, Sugiyama K. Establishment of an animal model of sedation using epidural anesthesia that uses the tailflick test for evaluating local anesthetic effects in rats. Exp Anim. 2013; 62(2):137-144

37. Wang AT, Liang DS, Liu YJ, Qi XR. Roles of ligand and TPGS of micelles in regulating internalization, penetration and accumulation against sensitive or resistant tumor and therapy for multidrug resistant tumors. Biomaterials. 2015;53:160-172.

38. Muthu MS, Kutty RV, Luo Z, Xie J, Feng SS. Theranostic vitamin E TPGS micelles of transferrin conjugation for targeted co-delivery of docetaxel and ultra bright gold nanoclusters. Biomaterials. 2015;39: 234-248.

39. Ribeiro LN, Franz-Montan M, Breitkreitz MC, et al. Nanostructured lipid carriers as robust systems for topical lidocaine-prilocaine release in dentistry. Eur J Pharm Sci. 2016;93:192-202.

40. Yu W, Liu C, Ye J, Zou W, Zhang N, Xu W. Novel cationic SLN containing a synthesized single-tailed lipid as a modifier for gene delivery. Nanotechnology. 2009;20(21):215102.

41. Zhai Y, Yang X, Zhao L, Wang Z, Zhai G. Lipid nanocapsules for transdermal delivery of ropivacaine: in vitro and in vivo evaluation. Int J Pharm. 2014;471(1-2):103-111.

42. Li TF, Fan H, Wang YX. Epidural sustained release ropivacaine prolongs anti-allodynia and anti-hyperalgesia in developing and established neuropathic pain. PLoS One. 2015;10(1):e0117321.

43. Danafar H, Davaran S, Rostamizadeh K, Valizadeh H, Hamidi M. Biodegradable m-PEG/PCL core-shell micelles: preparation and characterization as a sustained release formulation for curcumin. Adv Pharm Bull. 2014;4(Suppl 2):501-510.

44. Negi P, Singh B, Sharma G, Beg S, Katare OP. Biocompatible lidocaine and prilocaine loaded-nanoemulsion system for enhanced percutaneous absorption: QbD-based optimisation, dermatokinetics and in vivo evaluation. J Microencapsul. 2015;32(5):419-431.

45. Yokomizo Y, Sagitani H. Effects of phospholipids on the in vitro percutaneous penetration of prednisolone and analysis of mechanism by using attenuated total reflectance-Fourier transform infrared spectroscopy. J Pharm Sci. 1996;85(11):1220-1226.

46. Kwon SS, Kim SY, Kong BJ, et al. Cell penetrating peptide conjugated liposomes as transdermal delivery system of Polygonum aviculare L. extract. Int J Pharm. 2015;483(1-2):26-37.

47. Yue Y, Zhao D, Yin Q. Hyaluronic acid modified nanostructured lipid carriers for transdermal bupivacaine delivery: in vitro and in vivo anesthesia evaluation. Biomed Pharmacother. 2018;98:813-820.
Drug Design, Development and Therapy

\section{Publish your work in this journal}

Drug Design, Development and Therapy is an international, peerreviewed open-access journal that spans the spectrum of drug design and development through to clinical applications. Clinical outcomes, patient safety, and programs for the development and effective, safe, and sustained use of medicines are the features of the journal, which

\section{Dovepress}

has also been accepted for indexing on PubMed Central. The manuscript management system is completely online and includes a very quick and fair peer-review system, which is all easy to use. Visit http://www.dovepress.com/testimonials.php to read real quotes from published authors. 